\title{
Reconstruction and quantitative evaluation of porosity evolution during diagenesis -- implication for carbonate reservoir development
}

\author{
Leilei Yang', Linjiao Yu', Donghua Chen ${ }^{1}$, Changwei Li $^{2}$, Keyu Liu ${ }^{2}$, Zhuo Li ${ }^{1}$, \\ Xianglu Tang ${ }^{1}$, Zhiye Gao ${ }^{1}$, Dongdong Liu ${ }^{1}$, Wei Yang ${ }^{1}$
}
${ }^{1}$ Unconventional Petroleum Research Institute, China University of Petroleum, Beijing
${ }^{2}$ Department of Geosciences, China University of Petroleum, East China

Keywords: Diagenesis reconstruction; Fluid - rock interactions; Quantitative evaluation

Deep oil and gas reservoirs are becoming important substitute because of the successful explorations and huge resources. Conventional statistical inversion cannot accurately predict the distribution of deep reservoirs, which limits the exploration of deep oil and gas. Most previous studies about diagenetic facies, diagenetic sequence, diagenetic evolution and porosity prediction are mainly based on petrological test and sedimentology theory, lacking of quantitative research based on geochemical fluid rock interactions. However, it is well known that diagenesis is mainly completed in aqueous medium, and fluid - rock interactions basically determine porosity evolution.

In this study, typical carbonate reservoirs in the Tarim Basin, China are selected, and fluid - rock interactions during reservoir diagenesis are studied using qualitative calculation and forward - backward modelling. First, a series of petrological tests (e. g. $\mathrm{XRD}, \mathrm{SEM}$ and fluid inclusions) are conducted to qualitatively infer the diagenetic evolution process and typical diagenetic reactions. Second, diagenetic simulation stages are divided based on major diagenetic events. Then, fluid - rock interactions in each stage are simulated and all parameters are also corrected by comparing experimental data with simulation results. Finally, a reservoir diagenetic simulation system based on 
geochemical reactions is established, which is able to reconstruct the diagenetic evolution process at geological time scale and quantitatively redraw the porosity evolution curve. The comprehensive approach proposed in this study lead the theoretical innovation of quantitative research on diagenetic processes, which can provide support for the deep oil and gas exploration and development.

\section{Acknowledgments:}

This research is supported by the Natural Science Foundation of China (No. 41702249) and the Strategic Priority Research Program of the Chinese Academy of Sciences (No. XDA14010101). 